\title{
Kontinuierliches subkortikales Mapping zur Überwachung der Pyramidenbahn bei der Entfernung von motorisch eloquent gelegenen Hirntumoren
}

\section{Continuous Dynamic Mapping to Preserve the Corticospinal Tract During Surgery of Motor Eloquent Brain Tumors}

Autoren
Kathleen Seidel ${ }^{1}$, Philippe Schucht ${ }^{1}$, Jürgen Beck ${ }^{2}$, Andreas Raabe ${ }^{1}$

Institute

1 Inselspital, Department of Neurosurgery, Bern University Hospital, Bern, Switzerland

2 Department of Neurosurgery, University of Freiburg, Freiburg

\section{Schlüsselwörter}

Elektrische Stimulation, Intraoperatives Neuromonitoring, Motorisch Evozierte Potentiale, Pyramidenbahn,

Subkortikale Stimulation

Key words

Corticospinal tract, electrical stimulation, intraoperative neuromonitoring, motor evoked potential, subcortical mapping

\section{Bibliografie}

DOI https://doi.org/10.1055/a-1009-5386

Online-Publikation: 7.11.2019

Klin Neurophysiol 2019; 50: 220-226

(c) Georg Thieme Verlag KG Stuttgart · New York

ISSN 1434-0275

Korrespondenzadresse

Kathleen Seidel, MD

Inselspital

Universitätsklinik für Neurochirurgie

Universitätsspital Bern

3010 Bern

Schweiz

Kathleen.Seidel@insel.ch

\section{ZUSAMMENFASSUNG}

Die moderne neurochirurgische Onkologie verfolgt das Konzept der maximalen Tumorentfernung mit gleichzeitiger Funktionserhaltung. Dies ist bei Gliomen eine Herausforderung, da Tumorgrenzen nicht immer sichtbar sind und anatomische Landmarken verschoben sein können. Im folgenden Artikel werden intraoperative neurophysiologische Methoden zur Lokalisation und Überwachung des Primär Motorischen Kortex und der Pyramidenbahn in der supratentoriellen Tumorchirurgie diskutiert.

\section{ABSTRACT}

In modern neuro-oncological surgery, the key concept is maximal but safe tumor resection. However, this is a surgical challenge as tumor borders may be invisible, and anatomical landmarks might be distorted. In this review, we discuss intraoperative neurophysiological monitoring and mapping methods to identify the primary motor cortex and corticospinal tract in supratentorial tumor surgery.

\section{ABKÜRZUNGEN}

GTR gross total resection

MEP Motorisch evoziertes Potenzial

\section{Einleitung - das Neuroonkologische Dilemma}

Während der Operation von Hirntumoren werden 2 Konzepte verfolgt: das Ausmass der Resektion, welches mit dem Überleben kor- reliert, und die Vermeidung von neuen neurologischen Defiziten, was sich in der Lebensqualität wiederspiegelt [1,2]. Deshalb sollte die Tumorentfernung nicht nur nach anatomischem Wissen sondern funktionsgeleitet erfolgen [1-6].

Eine komplette Resektion oder fast komplette Resektion «gross total resection (GTR)» bleibt der Goldstand für die meisten intrakraniellen Tumoroperationen und die Evidenz hierfür steigt für sowohl höhergradige als auch niedriggradige Gliome [7-9]. In den präoperativen MRI Bildern werden bis zu $50 \%$ aller Hirntumoren 
fälschlicherweise als "eloquent» und damit als inoperabel eingeschätzt $[1,2,10]$. Intraoperatives Mapping dient also nicht nur dazu, wichtige eloquente Bahnen zu finden und die Funktion zu erhalten, sondern hilft auch zu verifizieren, ob ein vor der Operation als eloquent angenommener Tumor intraoperativ wirklich eloquent liegt [1].

Wenn man aber eine komplette Tumorentfernung anstrebt, ist oft der letzte und tiefste Punkt des Tumors in der Nähe von eloquenten Bahnen und so eine Verletzung dieser und ein bleibendes neurologisches Defizit eine Gefahr. So kann der neuroonkologische Vorteil einer kompletten Tumorentfernung [7, 9-13] durch ein neues neurologisches Defizit gemindert werden [14]. Deshalb ist das wichtige Konzept eine maximale aber sichere Tumorentfernung und dieses kann durch intraoperative neurophysiologische Methoden geleitet werden. In einer Metaanalyse von 90 Studien mit 8091 Patienten konnte gezeigt werden, dass intraoperatives Mapping bei Operationen von eloquent gelegenen Gliomen die späte neurologische Defizitrate von 8,2 auf 3,4\% reduzieren kann und gleichzeitig den Prozentsatz der "gross total resection" von 58 auf $75 \%$ verbessert [2].

Für diese funktionell geleitete neurochirurgische Operationstechnik gibt es 2 neurophysiologische Methoden: das Mapping und das Monitoring.

Elektrisches kortikales Mapping wurde bereits früh zur Identifizierung eloquenter kortikaler Areale etabliert [15-18]. Später wurde das subkortikale Mapping eingeführt, um wichtige Bahnen wie die Pyramidenbahn intraoperativ lokalisieren zu können [24, 19-22]. Eine gleichzeitig intraoperativ kontinuierliche Überwachung (Monitoring) evozierter Potenziale wie z. B. der motorisch evozierten Potenziale (MEP) erlaubt eine Echtzeit-Rückmeldung über die Integrität und Intaktheit des funktionellen Systems [5, 6, 23-26]. Beide Methoden, Mapping und Monitoring, sind weit verbreitet und haben die Sicherheit von Tumoroperationen deutlich verbessert [1, 5, 16, 20, 22, 23, 26-28]. Im folgenden Artikel werden Mapping-Techniken zum Aufsuchen der Pyramidenbahn diskutiert.

\section{Anästhesie}

Mapping zur Lokalisation motorischer Areale kann im Rahmen einer Wachoperation $[2,15,29,30]$ oder für die primär motorische Funktion und die Pyramidenbahn unter Allgemeinanästhesie durchgeführt werden [4-6, 28, 31].

Für die Allgemeinanästhesie ist die Standardempfehlung eine total Intravenöse Anästhesie mittels Propofol und Remifentanyl $[32,33]$. Es kann aber auch eine geringe Dosis an inhalativen Anästhetika in ausgewählten Fällen beigemischt werden. Kurz wirksame Muskelrelaxantien sollten nur zur Intubation verabreicht werden und anschliessend sollte eine Erholung mit der "train-of-four» Stimulation getestet werden [32].

\section{Die Technischen Hintergründe}

Konstant-Strom Stimulatoren sind sicherer und zuverlässiger für das intraoperative Mapping als Konstant-Spannungsstimulatoren, denn sie funktionieren unabhängig von der Oberflächenimpedanz des Gewebes [29]. Bei der Verwendung von Konstant-Spannung
Stimulatoren hängt der ausgegebene Strom von der Gewebeimpedanz ab. Dies kann nicht nur die Sicherheit deutlich beeinträchtigen, sondern ist ein zusätzliches Problem, wenn Stimulationsintensitäten mit dem Abstand, z. B. der Pyramidenbahn, ins Verhältnis gesetzt werden sollen; hier sollten deshalb Konstant-Strom Stimulatoren bevorzugt werden [6].

Klassische Stimulationspulse sind rechteckig und entweder monophasisch oder biphasisch [29]. Für das kortikale Mapping wird eine anodale Stimulation und für das subkortikale Mapping eine kathodale Stimulation empfohlen [34-29].

\section{Das Stimulationsparadigma}

Für das intraoperative Mapping werden hauptsächlich 2 Paradigmen verwendet. Die klassische Penfield-Technik verwendet eine Frequenz von 50 oder $60 \mathrm{~Hz}$, meistens eine Pulsbreite von $1 \mathrm{~ms}$ und eine Applikationsdauer von bis zu 4 Sekunden [15-17, 20]. Unter Anästhesie ist die ausgelöste Antwort eine tonische Muskelkontraktion, welche (abhängig von der Stimulationsintensität) nach wenigen Millisekunden beginnt und deren Amplitude mit der Stimulusdauer wächst $[4,35,36]$. Dies kann die Bestimmung der motorischen Schwelle deutlich erschweren [32].

Ein anderes, eher neueres Konzept ist die «short train» oder auch Hochfrequenzstimulation. Normalerweise sind dies 4-9 monophasische rechteckige Pulse mit einer Pulsbreite von $0,2-0,5 \mathrm{~ms}$ und einem Inter-Stimulus-Intervall von 2-4 ms (entsprechend 250 $500 \mathrm{~Hz}$ ), sowie einer Wiederholfrequenz bis zu $2 \mathrm{~Hz}[5,19,24,33$, 37]. Unter Anästhesie triggert die zeitliche Summation mehrerer absteigender Pulse letztendlich die MEP Antwort [24] mit einer definierten und einfach zu messenden Amplitude [6, 32, 35]. Deshalb ist zur Bestimmung von motorischen Schwellen der Reizantworten die «short-train» Stimulation der Penfield Stimulation überlegen $[4,6,19,32]$. Quantifizierbare Warnkriterien können einfacher etabliert und verglichen werden.

Wenn man die Ladung betrachtet, die beide Paradigmen applizieren, dann wird offensichtlich, wieso die Penfield Stimulation eine höhere intraoperative Anfallsrate auslöst als die "short train» Stimulation [38]. Für die Penfield Stimulation sind intraoperative Anfälle von 5-20\% beschrieben [17, 20, 36-38]. Im Gegensatz dazu liegen bei der "short train" Stimulation die Raten bei 1-4\% [5, 37-39].

In einer kürzlich publizierten Studie wurde gezeigt, dass in bestimmten Fällen, also Patienten mit einer schweren Epilepsie, Tumorinfiltration der Pyramidenbahn oder präoperativen Strahlentherapie, die Penfield Stimulation scheitern kann und nur die "short train" Stimulation zuverlässige Antworten auslöst [4].

\section{Die Auswahl der Stimulationssonde}

Es gibt verschiedene Mappingsonden. Die am häufigsten verwendeten Stimulationssonden sind bipolare Sonden, meistens mit 2 sphärischen Elektroden mit einem Abstand von 5 mm [15, 17]. Diese Sonden sind sehr selektiv und aktivieren besonderes das Gewebe unter den beiden Elektroden. Ist die gesuchte Struktur - z. B. die Pyramidenbahn - weiter vom Stimulationsort entfernt, dann wird das elektrische Feld weniger homogen und die Auslösung einer Reizantwort unwahrscheinlicher $[6,19,32]$. 
Eine alternative Möglichkeit ist die Stimulation mit einer Monopolaren Sonde [40]. Hier ist die Stimulationssonde selber die aktive Elektrode und der Strom fliesst zu einer weiter weg platzierten Referenzelektrode [41]. Das so generierte radiäre elektrische Feld ermöglicht, dass der Strom senkrecht das Axon aktiviert und so eine effektivere Stimulation auslöst [19]. Diese monopolaren Stimulationssonden sind zuverlässiger, wenn man mit quantitativen motorischen Schwellenwerten arbeiten möchte und versucht eine Distanz zur Pyramidenbahn abzuschätzen $[6,41]$.

Sind beide Sonden (bipolar und monopolar) mit derselben Intensität und demselben Stimulationsparadigma eingestellt, dann

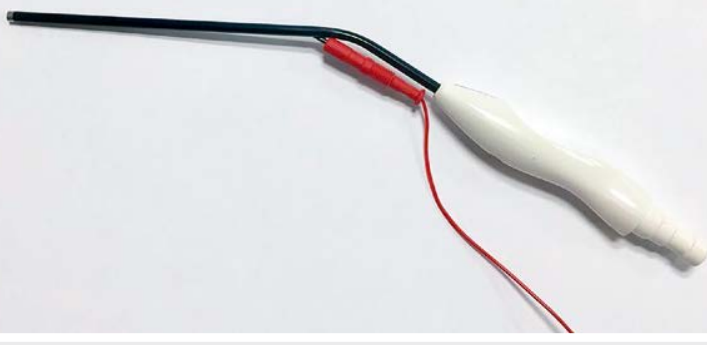

Abb. 1 Dynamischer Mapping Sauger. Die Sauger-Spitze enthält gleichzeitig eine monopolare Stimulationssonde. Der Stimulationssauger wird mittels eines Konnektors am Griff verbunden. Der Sauger ist elektrisch isoliert bis auf die Spitze. Der Sauger kann für die für subpiale Dissektion benutzt werden und gleichzeitige Stimulation zu jedem Zeitpunkt und an jeder Stelle der Tumorresektion. Quelle: Neurochirurgie Inselspital, Bern. ist die monopolare Stimulation sensitiver mit einer frühen Antwort, aber weniger fokalen Stimulation. Eine bipolare Stimulation ist spezifischer und selektiver, aber es sollte beachtet werden, dass eine Reizantwort nur dann ausgelöst wird, wenn die Sonde bereits sehr nah an der aufzusuchenden Struktur ist.

Die traditionelle Kombination ist eine bipolare Sonde mit dem Penfield Paradigma und die monopolare Stimulation mit dem «short train» Paradigma, aber auch andere Kombinationen sind möglich $[4,19]$.

\section{Subkortikales Mapping und der Abstand zur Pyramidenbahn}

Wenn intraoperatives Mapping dazu genutzt wird, um eine maximale aber sichere Tumorentfernung zu ermöglichen [1], dann ist eine wichtige Frage, wie weit die Pyramidenbahn vom aktuellen Resektionsort und damit aktuellen Stimulationsort entfernt ist.

Die Auslösung einer Reizantwort hängt von der Ladung ab, welche wiederum mit der Stimulationsintensität und Pulsbreite korreliert [19]. Und die Stromdichte vermindert sich mit der Entfernung vom Stimulationsort. Dies bedeutet, dass mit höherer Stimulationsintensität grössere Gewebeareale durchdrungen werden und vice versa [6]. Dies bedeutet, dass mit höherer Stimulationsintensität MEP Antworten bereits ausgelöst werden können, obwohl die Pyramidenbahn noch weiter entfernt ist. Mit geringerer Stimulationsintensität werden MEP nur in der näheren Umgebung ausgelöst. Dieses Verhältnis zwischen Stimulationsintensität und Distanz der Sonde zur Pyramidenbahn wurde von vielen Arbeitsgruppen

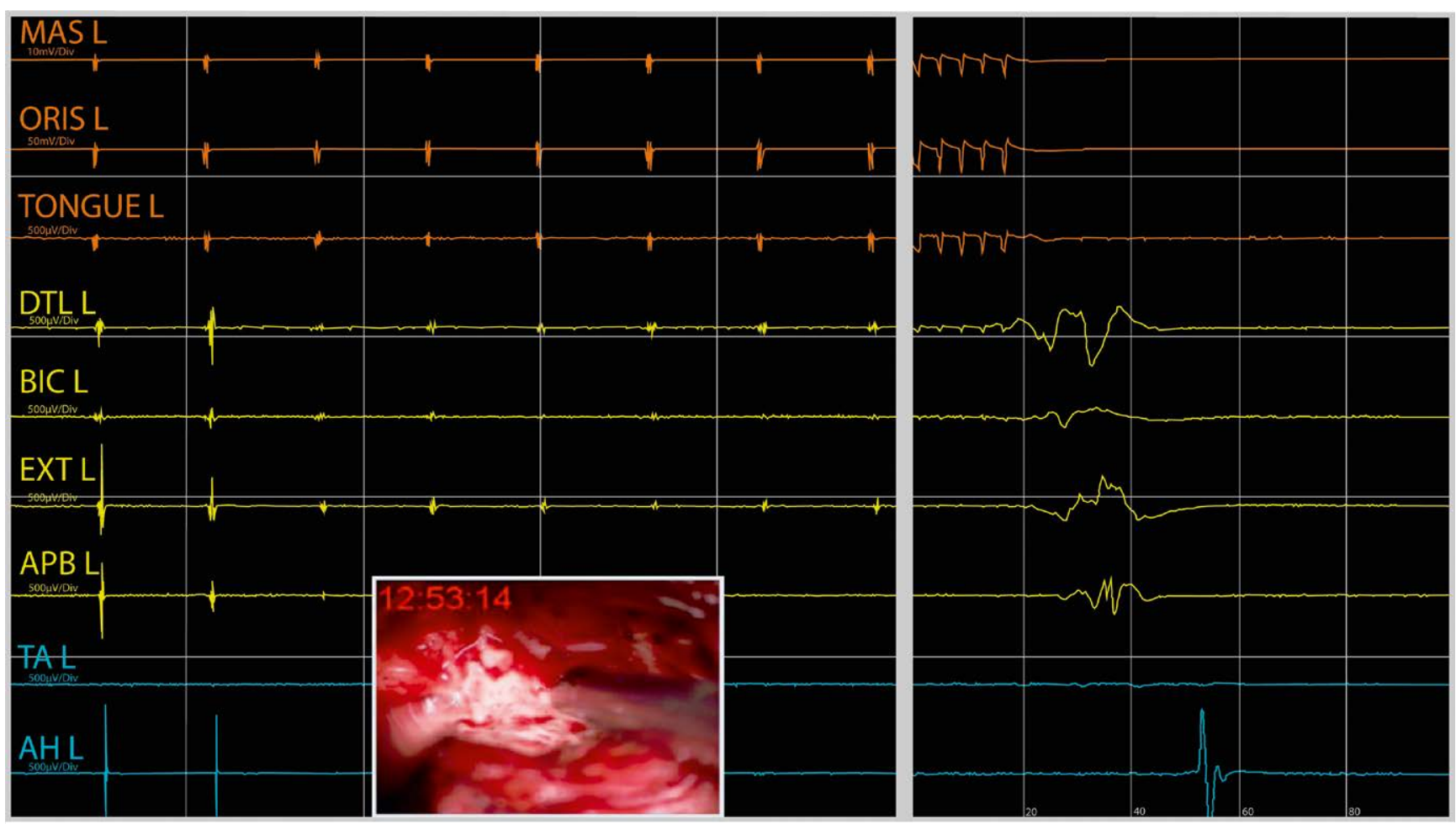

- Abb. 2 Mapping-Fenster. Freilaufendes EMG-Fenster (links) und getriggertes EMG Fenster (rechts). Ein akustischer Alarm Ton wird ausgelöst, sobald eine getriggerte MEP Antwort eine definierte Amplitude übersteigt. Die Stimulationsintensität kann manuell schrittweise gemindert werden, wenn die Resektionsgrenze sich der Pyramidenbahn nähert. In dieser Abbildung Gesichtsmuskeln (orange), Armmuskeln (gelb) und Bein (blau). Quelle: Neurochirurgie Inselspital, Bern. 


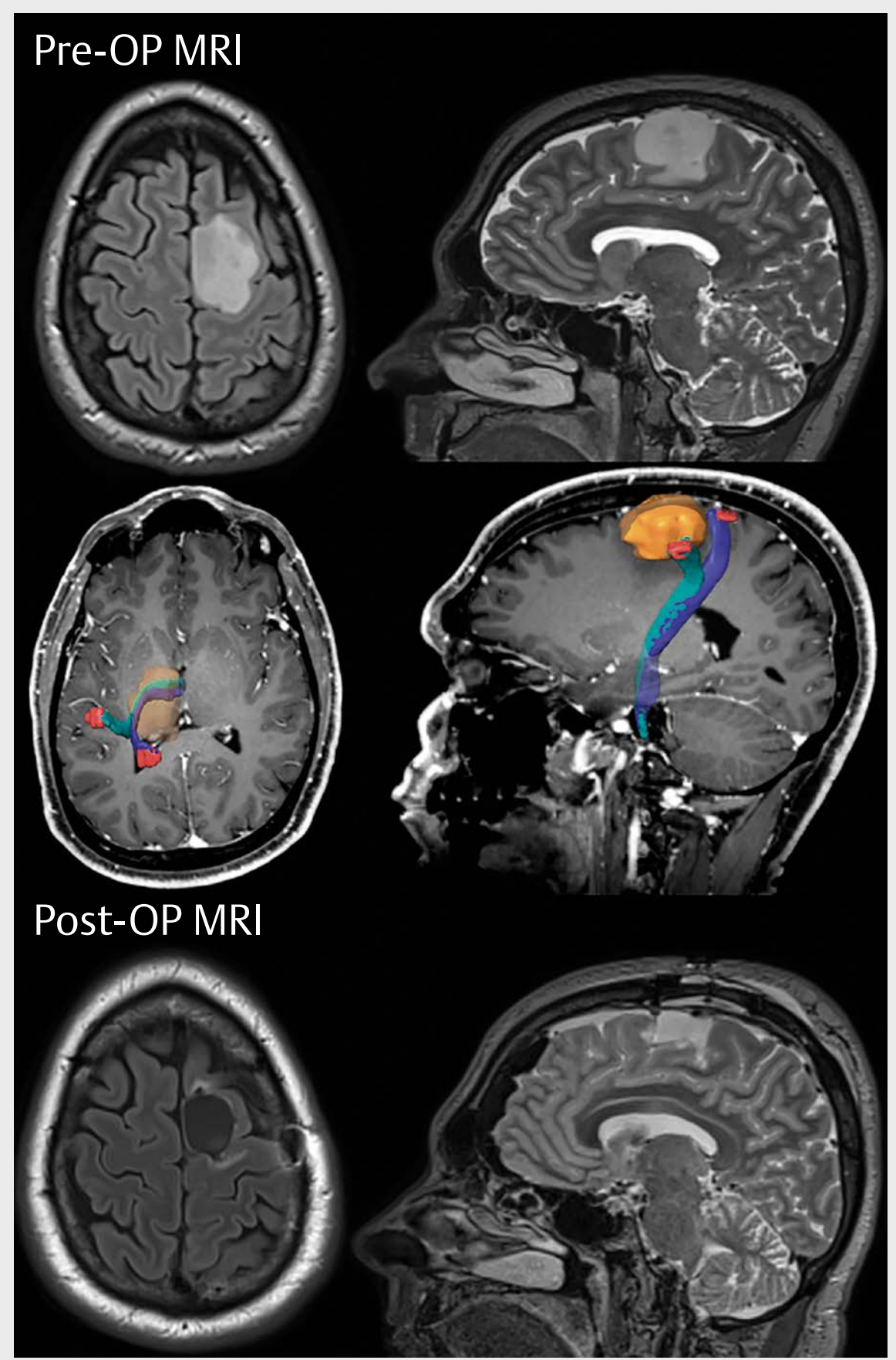

- Abb. 3 Fallbeispiel MRI. Oben: präoperative axiale FLAIR und sagittale T2 Schicht eines Tumors im superioren und medialen Gyrus frontalis. Mitte: Darstellung der Pyramidenbahn (DTI Fiber tracking mit navigierten TMS Punkten als Startpunkt) im Verhältnis zum Tumor (da es sich hier um eine Navigations-Bildgebung handelt, sind die Seiten invertiert). Unten: postoperative axiale FLAIR und sagittale T2 Schicht welche eine komplette Resektion eines Lower Grade Gliom (Oligodendriogliom WHO II LOH 1p/19q) zeigen. Der Patient hatte direkt nach der Operation ein SMA Syndrom der supplementär motorischen Areale (SMA), welches innerhalb eines Monates vollständig reversibel war. Zum intraoperativen Mapping und Monitoring siehe > Abb. 4. Quelle: Neurochirurgie Inselspital, Bern.

untersucht, um einen MEP Schwellenwert (in $\mathrm{mA}$ ) mit der Entfernung (in mm) zur Pyramidenbahn zu korrelieren [42-46].

Wenn man alle diese verschiedenen Studien vergleicht, dann ist darauf zu achten, dass z. T. verschiedene Stimulationssonden, eine unterschiedliche Anzahl von Pulsen im Train und unterschiedliche Pulsbreiten sowie Polaritäten verwendet wurden. So kann es rat- sam sein, eine definierte Pulsbreite und Anzahl von Stimuli zu definieren und subkortikal monopolar kathodal zu stimulieren [3,6, 31,32]. Bis jetzt konnte noch keine definitive Aussage zu diesem Abhängigkeitsverhältnis gemacht werden, aber die vage Faustregel «1 mA korreliert mit $1 \mathrm{~mm}$ » wird zunehmend für kathodale monopolare «short train» Stimulation verwendet. 


\section{Mapping Alarmkriterien}

Bei welchem Stimulationswert (in $\mathrm{mA}$ ) sollte man nun mit der Tumorresektion aufhören? Verschiedene Studien haben gezeigt, dass mit tieferen Stimulationsintensitäten das Risiko der mechanischen Verletzung der Pyramidenbahn steigt [5, 6, 31, 44]. Unter Verwendung von subkortikaler monopolarer kathodaler "short train» Stimulation hatten wir in einer Studie diese Abhängigkeit genau untersucht und eine Wahrscheinlichkeitsbeziehung zwischen Verletzung der Pyramidenbahn und tiefster Stimulationsintensität aufgestellt. Wir konnten aber ebenfalls zeigen, dass Werte bis und unter $3 \mathrm{~mA}$ immer noch sicher sein können, vorausgesetzt direkt kortikal überwachte MEP sind stabil und subkortikales Mapping wird mit sehr hoher Häufigkeit wiederholt $[6,41]$. Es existiert also ein Sicherheitskorridor zwischen niedrigen Stimulationsschwellen und Verletzung der Pyramidenbahn und somit bleibenden postoperativen motorischen Defiziten $[6,41]$. Jedoch einen definierten tiefsten Mappingwert zum Aufhören der Tumorresektion festzulegen, hängt von vielen Faktoren ab, wie Tumorhistopathologie, geplantes Ziel der Resektion, Infiltration des Tumors in andere eloquente Areale und Art der Blutstillung - und deshalb variiert das klinisch interne Sicherheitslimit zwischen verschiedenen neurochirurgischen Zentren [3-6, 31, 43-46].

Konventionelles intermittierendes und punktuelles Mapping kann die Gefahr eines nicht ausreichenden zeitlichen und räumlichen "Abtastens" bewirken - und dann können permanente motorische Defizite durch eine mechanische Verletzung der Pyramidenbahn trotz moderaten und scheinbar sicheren Stimulationswerten auftreten [6]. Deswegen ist zu empfehlen, subkortikales Mapping nicht intermittierend - sondern kontinuierlich - direkt während der Tumorentfernung durchzuführen. Dies ist absolut notwendig, wenn sehr tiefe Stimulationswerte von/unter $3 \mathrm{~mA}$ in der direkten Nähe zur Pyramidenbahn verwendet werden.

\section{Kontinuierliches dynamisches Mapping}

Wir haben kürzlich ein neues Mapping-Protokoll beschrieben: Wir haben ein Instrument entwickelt, welches eine kontinuierliche subkortikale Stimulation ermöglicht, ohne dabei den chirurgischen Workflow der Tumorentfernung zu unterbrechen [3]. Dies wurde erreicht, indem wir eine monopolare Stimulationssonde in einen klassischen chirurgischen Sauger integriert haben ( $\bullet$ Abb. 1) [3]. Die positive MEP Antwort wurde mit einem Alarm-Ton verbunden, um ein EchtzeitFeedback für den Chirurgen zu ermöglichen ( $\bullet$ Abb. 2) [3]. In einer initialen Studie konnten wir die Sicherheit und Zuverlässigkeit der Methode bei hocheloquent gelegenen Tumoren demonstrieren - mit einer Rate von $3 \%$ bleibender motorischer Defizite (aufgrund einer vaskulären und nicht einer mechanischen Läsion der Pyramidenbahn) [3]. In einer weiteren Studie konnten wir bei Glioblastomen eine komplette Resektion des Kontrastmittel aufnehmenden Anteils (CRET) von $69 \%$ und eine permanente Defizitrate von $4 \%$ (vaskuläre Läsionen) trotz Tumornähe zur Pyramidenbahn zeigen [14].

Kürzlich wurde das Konzept des dynamischen Mapping ebenfalls verwendet - aber mit direkter Stimulation über den CUSA $[47,48]$. In sehr seltenen Fällen könnte dies mit Mapping Ergebnissen interferieren [49]. Eine Integration in einen klassischen Sauger erscheint aus unserem Standpunkt ergonomischer, da dann das kontinuierliche Mapping bei allen chirurgisch wichtigen Schritten

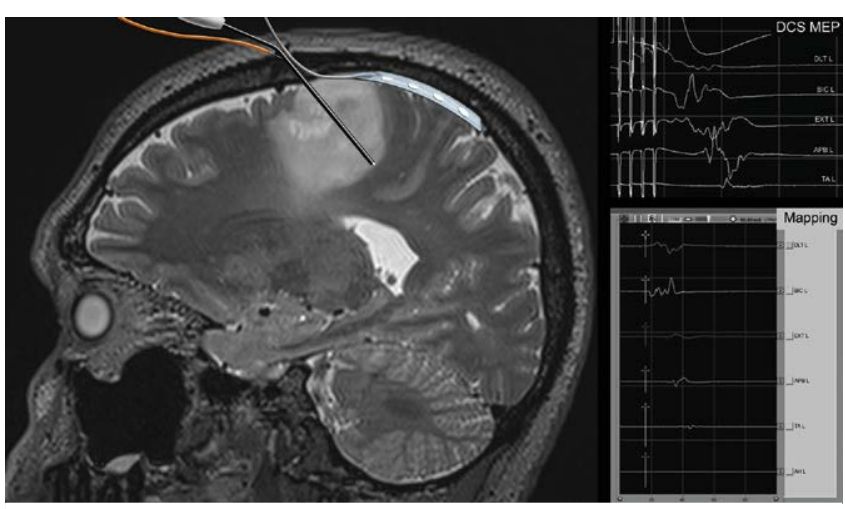

- Abb. 4 Fallbeispiel Neuromonitoring und Mapping-Konzept. Der Tumor ist anterior des Gyrus präzentralis lokalisiert. So kann eine Streifenelektrode zur direkten Stimulation und Überwachung der MEP platziert werden (DCS MEP). Die chirurgische Herausforderung liegt aber vielmehr in einem späteren Schritt. Sobald die Tumorresektion beginnt und in der Tiefe reseziert wird, ist keine subpiale Schicht mehr vorhanden und anatomische Landmarken helfen nicht viel weiter. Hier ist subkortikales Mapping essentiell, um den genauen Verlauf der Pyramidenbahn zu finden. Quelle: Neurochirurgie Inselspital, Bern.

inklusive subpialer Dissektion (mit verschiedenen Instrumenten) und Hämostase benutzt werden kann [3,14]. Eine Integration der Stimulationssonde in ein chirurgisches Instrument (CUSA oder Sauger) kann die Zuverlässigkeit, Akzeptanz und die ergonomische Handhabung des subkortikalen Mappings erhöhen ( $\triangleright$ Abb. $\mathbf{3}$ und -4) $[3,47-48]$.

\section{Mögliche Fallstricke}

Mapping kann nur Informationen über den Ort der Stimulation und distal davon angeben. Ist das Ziel eine Information über die komplette Integrität des primär motorischen Systems zu erhalten, dann werden zusätzlich MEP Monitoring-Methoden benötigt $[5,6,41]$. Ischämie z. B. durch unbeabsichtigte Koagulation eines GefässPerforators kann nur so erkannt werden [25, 26]. Deshalb sollten Mapping Techniken mit Monitoring Techniken kombiniert werden. Solange die MEP (v. a. direkt kortikal evozierte MEP) stabil sind, ist ein permanentes motorisches Defizit sehr unwahrscheinlich $[5,6,23,25,26,41]$. Der Nachteil ist, dass MEP Veränderungen plötzlich auftreten können und in zirka $40 \%$ der Fälle irreversibel sein können $[6,25,41]$.

\section{FAZIT}

Kontinuierliches subkortikales Mapping erlaubt eine Echtzeit Überwachung am Ort der Tumorresektion. Dies kann durch die Integration der Stimulationssonde in ein chirurgisches Instrument wie z. B. den Sauger erreicht werden. Mithilfe von der Variation der Stimulationsintensität kann der Abstand zur Pyramidenbahn abgeschätzt werden. Diese Methoden sind v. a. von Vorteil, wenn das Ziel ist, das Resektionsausmaß zu erhöhen und eine Tumorentfernung sehr nahe der Pyramidenbahn durchgeführt wird. Eine Analyse der Auswirkung auf das Langzeitüberleben der Patienten ist aber noch ausstehend. 


\section{Danksagung}

Wir danken Chantal Zbinden für die Erstellung der Abbildungen.

\section{Interessenskonflikt}

Der Mappingsauger wurde von 2 Autoren (AR und KS) entwickelt. Die Universität Bern erhält Royalities von Inomed. Die anderen Autoren haben keinen Interessenkonflikt zu Material und Methoden im Zusammenhang mit diesem Artikel.

\section{Literatur}

[1] Chang EF, Clark A, Smith JS et al. Functional mapping-guided resection of low-grade gliomas in eloquent areas of the brain: improvement of long-term survival. Clinical article. Journal of neurosurgery 2011; 114 : 566-573

[2] De Witt Hamer PC, Robles SG, Zwinderman AH et al. Impact of intraoperative stimulation brain mapping on glioma surgery outcome: a meta-analysis. Journal of clinical oncology: official journal of the American Society of Clinical Oncology 2012; 30: 2559-2565

[3] Raabe A, Beck J, Schucht P et al. Continuous dynamic mapping of the corticospinal tract during surgery of motor eloquent brain tumors: evaluation of a new method. Journal of neurosurgery 2014; 120 : 1015-1024

[4] Bello L, Riva M, Fava E et al. Tailoring neurophysiological strategies with clinical context enhances resection and safety and expands indications in gliomas involving motor pathways. Neuro-oncology 2014; 16: 1110-1128

[5] Sala F, Lanteri P. Brain surgery in motor areas: the invaluable assistance of intraoperative neurophysiological monitoring. Journal of neurosurgical sciences 2003; 47: 79-88

[6] Seidel K, Beck J, Stieglitz L et al. The warning-sign hierarchy between quantitative subcortical motor mapping and continuous motor evoked potential monitoring during resection of supratentorial brain tumors. Journal of neurosurgery 2013; 118: 287-296

[7] McGirt M], Chaichana KL, Gathinji M et al. Independent association of extent of resection with survival in patients with malignant brain astrocytoma. Journal of neurosurgery 2009; 110: 156-162

[8] McGirt M], Chaichana KL, Attenello F] et al. Extent of surgical resection is independently associated with survival in patients with hemispheric infiltrating low-grade gliomas. Neurosurgery 2008; 63: 700-707 author reply 707-708

[9] Lacroix M, Abi-Said D, Fourney DR et al. A multivariate analysis of 416 patients with glioblastoma multiforme: prognosis, extent of resection, and survival. Journal of neurosurgery 2001; 95: 190-198

[10] Stummer W, Pichlmeier U, Meinel T et al. Fluorescence-guided surgery with 5-aminolevulinic acid for resection of malignant glioma: a randomised controlled multicentre phase III trial. The lancet oncology 2006; 7: 392-401

[11] Jakola AS, Myrmel KS, Kloster R et al. Comparison of a strategy favoring early surgical resection vs a strategy favoring watchful waiting in low-grade gliomas. JAMA : the journal of the American Medical Association 2012; 308: 1881-1888

[12] Sanai N, Polley MY, McDermott MW et al. An extent of resection threshold for newly diagnosed glioblastomas. Journal of neurosurgery 2011; 115: 3-8

[13] Schucht P, Knittel S, Slotboom J et al. 5-ALA complete resections go beyond MR contrast enhancement: shift corrected volumetric analysis of the extent of resection in surgery for glioblastoma. Acta neurochirurgica 2014; 156: 305-312; discussion 312
[14] Schucht P, Seidel K, Beck J et al. Intraoperative monopolar mapping during 5-ALA-guided resections of glioblastomas adjacent to motor eloquent areas: evaluation of resection rates and neurological outcome. Neurosurgical focus 2014; 37: E16

[15] Penfield W. Somatic motor and sensory representation in the cerebral cortex of man as studied by electrical stimulation. Brain: a journal of neurology 1937; 60: 389-443

[16] Duffau H, Lopes M, Arthuis F et al. Contribution of intraoperative electrical stimulations in surgery of low grade gliomas: a comparative study between two series without (1985-96) and with (1996-2003) functional mapping in the same institution. Journal of neurology, neurosurgery, and psychiatry 2005; 76: 845-851

[17] Berger MS, Kincaid J, Ojemann GA et al. Brain mapping techniques to maximize resection, safety, and seizure control in children with brain tumors. Neurosurgery 1989; 25: 786-792

[18] Merton PA, Morton HB. Stimulation of the cerebral cortex in the intact human subject. Nature 1980; 285: 227

[19] Szelenyi A, Senft C, Jardan M et al. Intra-operative subcortical electrica stimulation: a comparison of two methods. Clinical neurophysiology: official journal of the International Federation of Clinical Neurophysiology 2011; 122: 1470-1475

[20] Duffau H, Capelle L, Denvil D et al. Usefulness of intraoperative electrical subcortical mapping during surgery for low-grade gliomas located within eloquent brain regions: functional results in a consecutive series of 103 patients. Journal of neurosurgery 2003; 98: 764-778

[21] Duffau $H$. The huge plastic potential of adult brain and the role of connectomics: new insights provided by serial mappings in glioma surgery. Cortex; a journal devoted to the study of the nervous system and behavior 2014; 58: 325-337

[22] Kombos T, Suss O, Vajkoczy P. Subcortical mapping and monitoring during insular tumor surgery. Neurosurgical focus 2009; 27: E5

[23] Deletis V, Camargo AB. Transcranial electrical motor evoked potential monitoring for brain tumor resection. Neurosurgery 2001; 49: 1488-1489

[24] Deletis V, Rodi Z, Amassian VE. Neurophysiological mechanisms underlying motor evoked potentials in anesthetized humans. Part 2. Relationship between epidurally and muscle recorded MEPs in man. Clinical neurophysiology: official journal of the International Federation of Clinical Neurophysiology. 2001; 112: 445-452

[25] Neuloh G, Pechstein U, Schramm J. Motor tract monitoring during insular glioma surgery. Journal of neurosurgery 2007; 106: 582-592

[26] Szelenyi A, Hattingen E, Weidauer S et al. Intraoperative motor evoked potential alteration in intracranial tumor surgery and its relation to signal alteration in postoperative magnetic resonance imaging. Neurosurgery 2010; 67: 302-313

[27] Krammer M], Wolf S, Schul DB et al. Significance of intraoperative motor function monitoring using transcranial electrical motor evoked potentials (MEP) in patients with spinal and cranial lesions near the motor pathways. British journal of neurosurgery 2009; 23: 48-55

[28] Neuloh G, Pechstein U, Cedzich C et al. Motor evoked potential monitoring with supratentorial surgery. Neurosurgery 2007; 61: 337-346 discussion 346-338

[29] Szelenyi A, Bello L, Duffau H et al. Intraoperative electrical stimulation in awake craniotomy: methodological aspects of current practice. Neurosurgical focus 2010; 28: E7

[30] Berger MS, Hadjipanayis CG. Surgery of intrinsic cerebral tumors. Neurosurgery 2007; 61: 279-304 discussion 304-275

[31] Plans G, Fernandez-Conejero I, Rifa-Ros X et al. Evaluation of the High-Frequency Monopolar Stimulation Technique for Mapping and Monitoring the Corticospinal Tract in Patients With Supratentorial Gliomas. A Proposal for Intraoperative Management Based on Neurophysiological Data Analysis in a Series of 92 Patients. Neurosurgery 2017; 81: 585-594 
[32] Seidel K, Beck J, Stieglitz L et al. Low-threshold monopolar motor mapping for resection of primary motor cortex tumors. Neurosurgery 2012; 71: 104-114 discussion 114-105

[33] Taniguchi M, Cedzich C, Schramm J. Modification of cortical stimulation for motor evoked potentials under general anesthesia: technical description. Neurosurgery 1993; 32: 219-226

[34] Hern JE, Landgren S, Phillips CG et al. Selective excitation of corticofugal neurones by surface-anodal stimulation of the baboon's motor cortex. The Journal of physiology 1962; 161: 73-90

[35] Kombos T, Suess O, Kern BC et al. Comparison between monopolar and bipolar electrical stimulation of the motor cortex. Acta neurochirurgica 1999; 141: 1295-1301

[36] Yingling CD, Ojemann S, Dodson B et al. Identification of motor pathways during tumor surgery facilitated by multichannel electromyographic recording. Journal of neurosurgery 1999; 91 : 922-927

[37] Macdonald DB, Skinner S, Shils J et al. Intraoperative motor evoked potential monitoring - a position statement by the American Society of Neurophysiological Monitoring. Clinical neurophysiology: official journal of the International Federation of Clinical Neurophysiology 2013; 124: 2291-2316

[38] Szelenyi A, Joksimovic B, Seifert V. Intraoperative risk of seizures associated with transient direct cortical stimulation in patients with symptomatic epilepsy. Journal of clinical neurophysiology: official publication of the American Electroencephalographic Society 2007; 24: $39-43$

[39] Ulkatan S, Jaramillo AM, Tellez M] et al. Incidence of intraoperative seizures during motor evoked potential monitoring in a large cohort of patients undergoing different surgical procedures. Journal of neurosurgery 2017; 126: 1296-1302

[40] Suess O, Kombos T, Hoell T et al. A new cortical electrode for neuronavigation-guided intraoperative neurophysiological monitoring: technical note. Acta neurochirurgica 2000; 142: 329-332
[41] Landazuri P, Eccher M. Simultaneous Direct Cortical Motor Evoked Potential Monitoring and Subcortical Mapping for Motor Pathway Preservation During Brain Tumor Surgery: Is it Useful? Journal of clinical neurophysiology: official publication of the American Electroencephalographic Society 2013; 30: 623-625

[42] Kamada K, Todo T, Ota T et al. The motor-evoked potential threshold evaluated by tractography and electrical stimulation. Journal of neurosurgery 2009, doi:10.3171/2008.9.JNS0841410.3171/ 2008.9.JNS08414 [pii]

[43] Nossek E, Korn A, Shahar T et al. Intraoperative mapping and monitoring of the corticospinal tracts with neurophysiological assessment and 3-dimensional ultrasonography-based navigation. Clinical article. Journal of neurosurgery 2011; 114: 738-746

[44] Prabhu SS, Gasco J, Tummala $S$ et al. Intraoperative magnetic resonance imaging-guided tractography with integrated monopolar subcortical functional mapping for resection of brain tumors. Clinical article. Journal of neurosurgery 2011; 114: 719-726

[45] Ohue S, Kohno S, Inoue A et al. Accuracy of diffusion tensor magnetic resonance imaging-based tractography for surgery of gliomas near the pyramidal tract: a significant correlation between subcortical electrical stimulation and postoperative tractography. Neurosurgery 2012; 70: 283-293 discussion 294

[46] Shiban E, Krieg SM, Haller B et al. Intraoperative subcortical motor evoked potential stimulation: how close is the corticospinal tract? Journal of neurosurgery 2015; 123 : 711-720

[47] Shiban E, Krieg SM, Obermueller T et al. Continuous subcortical motor evoked potential stimulation using the tip of an ultrasonic aspirator for the resection of motor eloquent lesions. Journal of neurosurgery 2015; 123: 301-306

[48] Roth J, Korn A, Bitan-Talmor Y et al. Subcortical Mapping Using an Electrified Cavitron UltraSonic Aspirator in Pediatric Supratentorial Surgery. World neurosurgery 2017; 101: 357-364

[49] Carrabba G, Mandonnet E, Fava E et al. Transient inhibition of motor function induced by the Cavitron ultrasonic surgical aspirator during brain mapping. Neurosurgery 2008; 63: E178-E179; discussion E179 